\title{
Photoresponsive Nanometer-Scale Iron Alginate Hydrogels: A Study of Gel-Sol Transition Using a Quartz Crystal Microbalance
}

\author{
Miryam Criado-Gonzalez, ${ }^{*, \dagger}, \oplus \odot$ Laura Corbella, ${ }^{\dagger}$ Bernard Senger, ${ }^{\S, \|}$ Fouzia Boulmedais, ${ }^{\ddagger \oplus}$ \\ and Rebeca Hernández* ${ }^{*} \odot$ \\ ${ }^{\dagger}$ Instituto de Ciencia y Tecnología de Polímeros (ICTP-CSIC), c/Juan de la Cierva, 3, 28006 Madrid, Spain
${ }^{\ddagger}$ Institut Charles Sadron, Université de Strasbourg, CNRS, UPR 22, 67034 Strasbourg, France
${ }^{\S}$ INSERM, UMR-S 1121, 11 rue Humann, 67085 Strasbourg, France
${ }^{\|}$Faculté de Chirurgie Dentaire, Université de Strasbourg, 8 rue Sainte Elisabeth, 67000 Strasbourg, France
}

Supporting Information

ABSTRACT: Alginate $/ \mathrm{Fe}^{3+}$ hydrogels were fabricated on hyaluronic acid (HA) and poly(allylamine hydrochloride) (PAH) multilayers to yield photoresponsive nanometer-scale hydrogels. Light irradiation of the resulting hydrogels induced the photoreduction of "hard" $\mathrm{Fe}^{3+}$ to "soft" $\mathrm{Fe}^{2+}$ cations, leading to changes in the mechanical properties of the hydrogels related to their crosslinking behavior. The buildup and the phototriggered response of the supported alginate hydrogels were followed in situ with a quartz crystal microbalance (QCM) using an open cell allowing light irradiation from an LED source on top of the hydrogel. The results were correlated to the release profiles of folic acid, employed herein

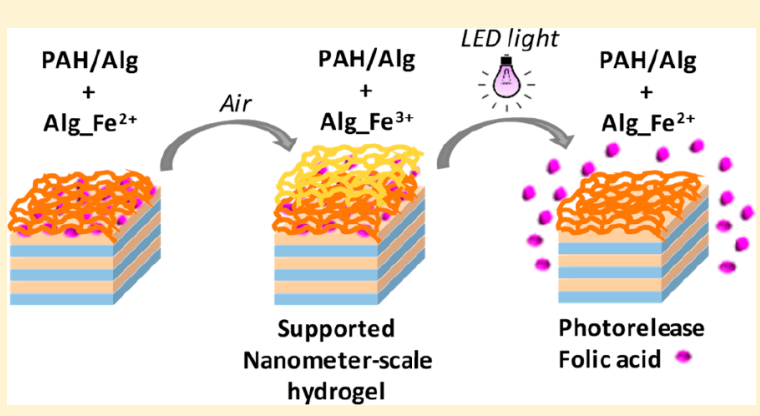
as a drug model, obtained from light-irradiated supported iron alginate hydrogels.

\section{INTRODUCTION}

The design of novel functional biomaterials is nowadays focused on the employment of biobased polymers capable of responding to external stimulus, such as magnetic fields, ${ }^{1-3}$ temperature, ${ }^{4-6} \mathrm{pH}^{7,8}$ light, ${ }^{9,10}$ electrochemical potentials, ${ }^{11}$ and mechanical signals ${ }^{12,13}$ in a well-controlled manner. ${ }^{14}$ Stimuli-responsive hydrogels are widely employed in different applications such as drug delivery, ${ }^{15}$ tissue engineering, ${ }^{16}$ sensors, ${ }^{17}$ actuators, ${ }^{18}$ and energy storage. ${ }^{19}$ Biopolymers are receiving increasing attention in engineering and biomedical fields due to their inherent properties, such as biocompatibility, biodegradability, and nontoxicity. ${ }^{20}$ Alginate (Alg) is a polysaccharide isolated from brown seaweeds or certain bacteria, that consists of $\beta$ - $(1,4)$-linked D-mannuronic acid (M) and $\alpha$-L-guluronic acid (G) residues arranged in blocks of $M$ fragments, $G$ fragments, and alternating $G$ and $M$ fragments. Subject to ongoing research, ${ }^{21}$ alginate is able to form hydrogels by ionotropic gelation through interactions of $\mathrm{G}$ fragments with divalent and multivalent ions. ${ }^{22}$ One of the most employed cations to cross-link sodium alginate is $\mathrm{Ca}^{2+23}$ although transition metal ions, such as ferric ions $\left(\mathrm{Fe}^{3+}\right)$, have also been reported as cross-linker ions for the formation of alginate hydrogels. ${ }^{24}$ The coordination bonds between G fragments and $\mathrm{Fe}^{3+}$ ions create a covalent contribution not seen with $\mathrm{Ca}^{2+}$ ions which is attributed to the dynamic nature of the metal-coordination bond. This covalent link allows extra functionality by changing the metal-coordination environment, giving rise to new stabilities and mechanical properties for the resulting alginate hydrogels. ${ }^{25,26} \mathrm{Fe}^{3+}$ ions can be subjected to a photochemical reduction triggered by visible-light leading to ferrous ions $\left(\mathrm{Fe}^{2+}\right)$, and this phenomenon has been employed to induce the gel-sol transition of bulk alginate/ $\mathrm{Fe}^{3+}$ hydrogels. $9,27 \mathrm{Fe}^{2+}$ is a "soft" metal cation with lower ability than "hard" $\mathrm{Fe}^{3+}$ cations for cross-linking alginate affecting the alginate gel stability. ${ }^{28}$ The reduction of $\mathrm{Fe}^{3+}$ to $\mathrm{Fe}^{2+}$ can also be achieved electrochemically on an electrode by application of reductive potentials. ${ }^{29}$ As an example, iron-cross-linked poly(acrylic acid) hydrogels have been obtained through sol-gel transition using $\mathrm{Fe}^{2+} / \mathrm{Fe}^{3+}$ redox couple and application of an external stimuli such as light, air oxidation, ${ }^{30}$ or an applied potential. ${ }^{31}$

In the past few decades, layer-by-layer ( $\mathrm{LbL}$ ) assembly, based on the sequential adsorption of polycations and polyanions over a substrate, has been studied as a prevalent method for the fabrication of nanoscale structures due to its simplicity and versatility. ${ }^{32}$ Multilayer thin films built up through LbL assembly have been widely employed in the biomedical field for controlled and sustained drug delivery applications because of its ability to safely load drugs and release them in a controlled manner, improving the drug efficacy. ${ }^{33-35}$ We report herein the synthesis and extensive characterization of a unique class of iron alginate hydrogels,

Received: April 26, 2019

Revised: August 5, 2019

Published: August 6, 2019 
Scheme 1. Fabrication of Supported Nanometer-Scale Alginate Hydrogels by LbL Dipping
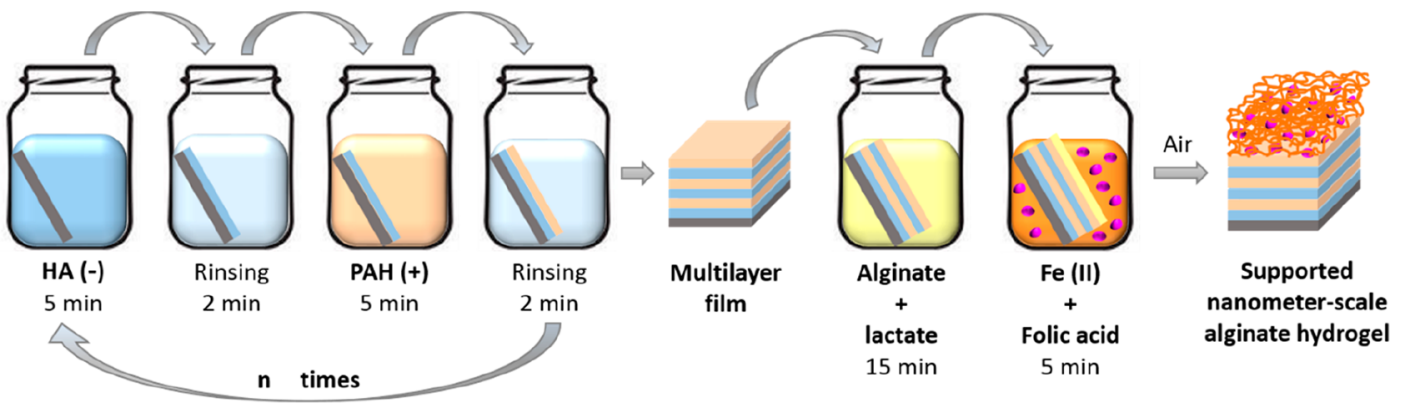

supported on a multilayer film, with light-responsive behavior. To that aim, alginate hydrogels cross-linked with iron ions were fabricated on top of multilayer films of hyaluronic acid (HA) and poly(allylamine hydrochloride) (PAH). To the best of our knowledge, this is the first time that the formation and photodissolution of nanometer-scale iron alginate hydrogels has been studied and followed in situ with a quartz crystal microbalance with dissipation (QCM-D) opening the route for the formation of new materials combining multilayer films and gels at the nanometer scale. Finally, folic acid was employed as a model drug to demonstrate the concept of phototriggered release from supported iron alginate hydrogels.

\section{EXPERIMENTAL SECTION}

Materials. Sodium alginate (Alg) was supplied by Sigma-Aldrich (A2158, lot 090M0092V). According to the fabricant, viscosity was $136 \mathrm{cps}\left(2 \% \mathrm{w} / \mathrm{v}\right.$ in water at $\left.25^{\circ} \mathrm{C}\right)$. Molecular weight $\left(M_{\mathrm{w}}\right)$ was 166 $\mathrm{kDa}$, as determined by capillary viscosimetry at $25^{\circ} \mathrm{C}$ using as solvent $0.1 \mathrm{M}$ sodium chloride and applying the equation of Mark-Houwink $\left(k=2 \times 10^{-5} \mathrm{dL} / \mathrm{g}, a=1.0\right) .{ }^{36}$ Hyaluronic acid (HA), $M_{\mathrm{w}} 301-450$ $\mathrm{kDa}$ was purchased from Lifecore Biomedical. Poly(allylamine hydrochloride) (PAH) with a molecular weight of $58 \mathrm{kDa}$ was supplied by Aldrich (283223, lot MKBJ4277 V). Iron(II) chloride tetrahydrate $\left(\mathrm{Fe}^{2+}\right)$ and poly(ethylene imine) (PEI) with a molecular weight $\left(M_{\mathrm{w}}\right)$ of $25 \mathrm{kDa}$ were supplied by Aldrich. Sodium L-lactate, $98 \%$, was supplied by Alfa Aesar. The compounds were used as received. The folic acid was obtained from a commercial formulation called ACFOL that contains $5 \mathrm{mg}$ of folic acid per pill. The pill was dissolved in Milli-Q water and filtered first in a cotton-plugged Pasteur pipet and second in a syringe filter and diluted until reaching the desired concentration. The polyelectrolytes, PEI, HA, and PAH, were dissolved in $0.15 \mathrm{M} \mathrm{NaCl}$. The other compounds were dissolved in Milli-Q water.

Preparation and Characterization of Bulk Iron Alginate Hydrogels (Alg_Fe). Bulk Alg_Fe hydrogels were prepared by mixing, in a vial, $0.5 \mathrm{~mL}$ of an aqueous solution of $\mathrm{Alg}(5 \mathrm{or} 10 \mathrm{mg} /$ $\mathrm{mL}$ ) and $2.24 \mathrm{mg} / \mathrm{mL}$ of sodium lactate with $0.5 \mathrm{~mL}$ of an aqueous solution containing $\mathrm{Fe}^{2+}(4$ or $6 \mathrm{mg} / \mathrm{mL})$ and $1 \mathrm{mg} / \mathrm{mL}$ of folic acid. Gel formation was ascertained through the inverted vial test. Samples were denoted as $\operatorname{Alg} x$ Fey, were $x$ stands for the concentration of alginate in $\mathrm{mg} / \mathrm{mL}$ and $y$ stands for the concentration of $\mathrm{Fe}^{2+}$ in $\mathrm{mg} /$ $\mathrm{mL}$. Phototriggered gel dissolution was carried out by irradiation of the Alg_Fe gel with an LED source emitting $405 \mathrm{~nm}$ light at a laser power of $10.2 \mathrm{~W} / \mathrm{cm}^{2}$. Samples were located at a distance of approximately $3 \mathrm{~cm}$ from the LED source. The time to achieve gelsol transition was determined for bulk alginate hydrogels with 5 and $10 \mathrm{mg} / \mathrm{mL}$ alginate concentrations and 4 and $6 \mathrm{mg} / \mathrm{mL} \mathrm{Fe}^{2+}$ concentrations.

Preparation and Characterization of HA/PAH Multilayer Films. HA/PAH multilayers were built on silicon slides $(20 \times 40$ $\mathrm{mm}$, Siegert) previously cleaned with piranha solution $\left(3: 1 \mathrm{H}_{2} \mathrm{SO}_{4} /\right.$ $\mathrm{H}_{2} \mathrm{O}_{2}$ ) for $10 \mathrm{~min}$ and rinsed extensively with water. As the silicon substrate is negatively charged, a layer of PEI $(1 \mathrm{mg} / \mathrm{mL})$ was deposited as a first layer to get a homogeneous positively charged substrate. For that, substrates were immersed in a solution of PEI for $5 \mathrm{~min}$ and then in a solution of Milli- $\mathrm{Q}$ water for $2 \mathrm{~min}$. HA/PAH multilayer films were produced through sequential dipping of a silicon substrate into the HA (polyanion) and $\mathrm{PAH}$ (polycation) solutions for $5 \mathrm{~min}$ with a rinsing step in $0.15 \mathrm{M} \mathrm{NaCl}$ for $2 \mathrm{~min}$ after each polyelectrolyte deposition. Substrates were dried after each rinsing step. The cycle was repeated until the desired number of bilayers $(n)$ was obtained. Samples were denoted as $(\mathrm{HA} z / \mathrm{PAH} w)_{n}$, where $z$ and $w$ stand for the concentration of $\mathrm{HA}$ and $\mathrm{PAH}$ in $\mathrm{mg} / \mathrm{mL}$, respectively.

Measurements of $(\mathrm{HA} z / \mathrm{PAH} w)_{n}$ multilayer film thickness were carried out with a PLASMOS SD 2300 ellipsometer operating at the single wavelength of $632.8 \mathrm{~nm}$ and a constant angle of $70^{\circ}$. Due to the inherent limit of ellipsometry to simultaneously determine the refractive index and the film thickness for very thin films, the refractive index of all films was assumed to be constant at $n=1.465 .{ }^{8}$ While this procedure will lead to slightly incorrect values with respect to the absolute film thicknesses, it allows for the quick and precise determination of relative film thicknesses. Thickness values obtained with the assumption of a fixed refractive index for all films are of better precision than required for the comparison of film growth data as in this report. For each substrate studied, 10 different thickness measurements were randomly taken on different regions over an area of a few $\mathrm{cm}^{2}$ of the film surface.

The topography and surface roughness of $(\mathrm{HAz} / \mathrm{PAH} w)_{n}$ multilayer films were evaluated by atomic force microscopy (AFM) using a Veeco MultiMode scanning probe microscope (SPM) with a Nanoscope Iva controller employing a tip RTESP7 at a frequency of oscillation of $265-309 \mathrm{kHz}$. All measurements were performed in air, at room temperature, on films supported on silicon wafers. Samples were scanned over square regions of $50 \mu \mathrm{m}$. For thickness determination, a scratch was applied at the center of the film, leaving a part of the substrate exposed. The images were taken in an area of 50 $\times 50 \mu \mathrm{m}$ that comprises the scratch. For the characterization of the thickness, three different regions of the image were evaluated. The image processing was performed with the software Nanoscope 6.14 R1.

Fabrication of Supported Alginate Hydrogels on HA/PAH Multilayer Films. As a first step, a HA/PAH multilayer film of HA $(2.5 \mathrm{mg} / \mathrm{mL})$ and PAH $(2.0 \mathrm{mg} / \mathrm{mL})$ with a total number of bilayers of 10 was built as described previously and immersed into an aqueous solution of alginate $(5 \mathrm{mg} / \mathrm{mL})$ and sodium lactate $(2.24 \mathrm{mg} / \mathrm{mL})$ for $15 \mathrm{~min}$. Without rinsing, the sample was introduced in an aqueous $\mathrm{Fe}^{2+}$ solution $(4 \mathrm{mg} / \mathrm{mL})$ for $5 \mathrm{~min}$ and left to air-dry overnight. To encapsulate a drug (folic acid) inside the gel, $\mathrm{Fe}^{2+}$ was dissolved in an aqueous solution of folic acid $(1 \mathrm{mg} / \mathrm{mL})$. After the process, samples were left to dry at room temperature $\left(20^{\circ} \mathrm{C}\right)$ in a horizontal position for $12 \mathrm{~h}$ in a closed Petri dish of $60 \mathrm{~mm}$ diameter to ensure that the gel layer remains homogeneously distributed along the substrate surface. Three samples were measured for each condition under study to obtain the average and standard deviation. A schematic representation of the procedure is shown in Scheme 1.

Quartz Crystal Microbalance with Dissipation (QCM-D) Study. QCM-D experiments were carried out in an open QCM-D cell on a Q-Sense E1 apparatus (Q-Sense AB, Gothenburg, Sweden) 
a)

\section{Gel formation}

Inverted tube

test
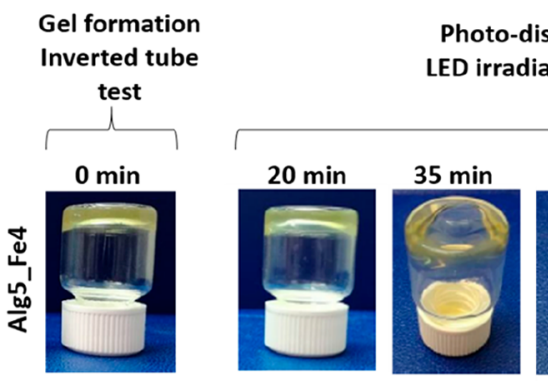

hoto-dissolution under

irradiation $(\lambda=405 \mathrm{~nm})$

b)
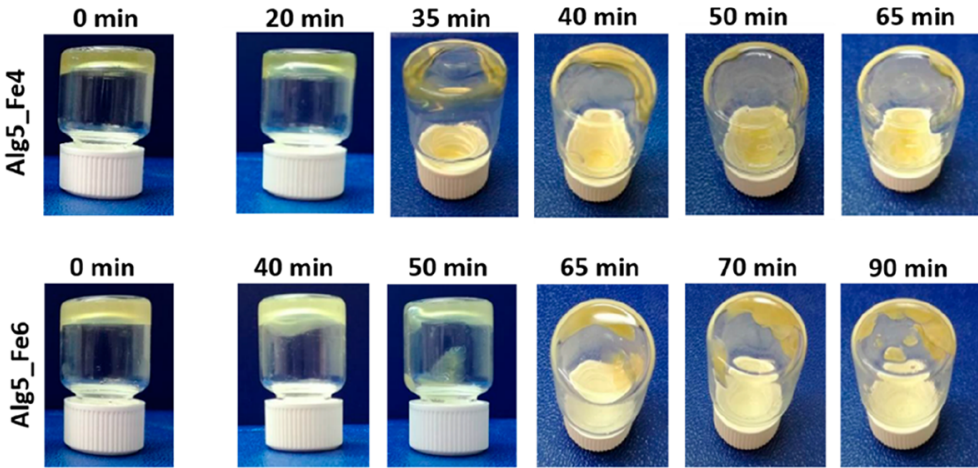

c)
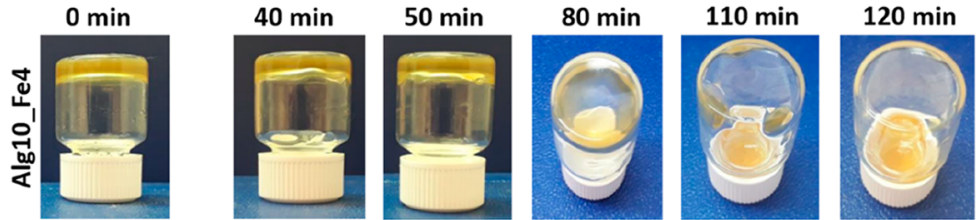

Figure 1. Gel formation (left) and photodissolution (right) of $\mathrm{Alg} \mathrm{Fe}$ hydrogels obtained at different concentrations of $\mathrm{Alg}$ and $\mathrm{Fe}^{2+}$ and fixed concentrations of sodium lactate and folic acid (a) Alg5_Fe4 after 24 h, (b) Alg5_Fe6 after 24 h, and (c) Alg10_Fe4 after 72 h. The gel dissolution was triggered by light irradiation.

at $22{ }^{\circ} \mathrm{C}$ by monitoring the resonance frequencies of the gold-coated crystal, as well as the dissipation factors at four frequencies, the fundamental frequency at $5 \mathrm{MHz}(\nu=1)$, and the third, fifth, and seventh harmonics $(\nu=3,5$, and 7$)$ at 15,25 , and $35 \mathrm{MHz}$, respectively. The QCM-D experiments give information on the adsorption process, as well as on viscoelastic properties of the adsorbed film. The results corresponding to changes in the frequency shifts $\left(\Delta f_{\nu} / \nu\right)$ and dissipation $\left(\Delta D_{\nu}\right)$, obtained from QCM-D experiments, were analyzed using the Voigt-Voinova model to determine the film thickness. The density was fixed at $1 \mathrm{~g} / \mathrm{cm}^{3}$ and the viscosity at $0.91 \mathrm{mPa} \cdot \mathrm{s}^{37}$

Phototriggered Drug Release. Folic acid release was determined from bulk and supported iron alginate hydrogels as follows: samples were introduced to a vial in $1 \mathrm{~mL}$ of Milli-Q water and irradiated with light from an LED source $(50 \mathrm{~mW}$ at $\lambda=405$ $\mathrm{nm}$ ). Aliquots of $0.5 \mathrm{~mL}$ were extracted and replaced by pure water at different times. All the aliquots were measured using NanoDrop One ${ }^{\mathrm{C}}$ (Thermo Scientific) at $\lambda=280 \mathrm{~nm}$. For comparison, the same protocol was applied to hydrogels not exposed to light.

\section{RESULTS AND DISCUSSION}

Multilayer films based on PAH and HA are developed to act as a support and seed layer for the fabrication of iron alginate hydrogels at the nanometer scale. The interaction between the multilayer film $\mathrm{HA} / \mathrm{PAH}$ and alginate is studied to analyze its effect in the phototriggered release of folic acid.

Bulk Iron Alginate Gel Formation and Phototriggered Gel-Sol Transition. We first optimized the experimental conditions (iron and alginate concentration) for alginate gel formation and its photodissolution in the bulk. The alginate gel formation in the presence of $\mathrm{Fe}^{2+}$ is a combination of ionotropic gelation and acid gelation. The ionotropic gelation is produced by the interaction of an ionic polymer with an oppositely charged cross-linker and the acid gelation is the regular way of gelation of alginic acid salts. In this case, the interactions will occur between free $\mathrm{COO}^{-}$groups of alginate and $\mathrm{Fe}^{2+}$ ions used as cross-linker. ${ }^{22} \mathrm{Fe}^{2+}$ cations have a limited ability to cross-link alginate, giving rise to "soft" hydrogels. In our case, $\mathrm{Alg} \_\mathrm{Fe}^{2+}$ gels were put in contact with air to induce the oxidation into $\mathrm{Fe}^{3+}$, leading to the formation of "hard" hydrogels. To choose the best concentration of alginate and iron to form a gel, different concentrations of $\mathrm{Alg}$ ( 5 and 10 $\mathrm{mg} / \mathrm{mL}$ ) and $\mathrm{Fe}^{2+}(4$ and $6 \mathrm{mg} / \mathrm{mL}$ ) were tested with a fixed concentration of sodium lactate $(2.24 \mathrm{mg} / \mathrm{mL})$. Figure 1 (left) shows the images corresponding to the inverted vial test of gels Alg5_Fe4 (Figure 1a) and Alg5_Fe6 (Figure 1b) obtained after $24 \mathrm{~h}$ at room temperature. The sample Alg10_Fe4 (Figure 1c) gelled after $72 \mathrm{~h}$ at room temperature according to the inverted vial test. In the absence of $\mathrm{Fe}^{2+}$ ions, the gelation did not take place (Figure S1).

Figure 1 (right) shows the photodissolution of Alg_Fe hydrogels in the presence of an LED source $(\lambda=405 \mathrm{~nm})$. $\mathrm{Fe}^{3+}$ cations are reduced to $\mathrm{Fe}^{2+}$ through photochemical reaction, inducing the dissolution of the gel. Sodium lactate was chosen as photoreducing agent to enhance the dissolution of the gel thanks to its oxidative decarboxylation concomitant with $\mathrm{Fe}^{3+}$ reduction. ${ }^{38,39} \mathrm{Alg} 5$ _Fe4 gel was completely dissolved after light irradiation for $65 \mathrm{~min}$ in contrast to Alg5_Fe6 gel, obtained with a higher quantity in $\mathrm{Fe}^{2+}$, for which some lumps of gel remained in suspension after light irradiation for $90 \mathrm{~min}$ as shown in Figure $1 \mathrm{~b}$. Likewise, the sample Alg10_Fe4, in which the alginate concentration was increased up to $10 \mathrm{mg} / \mathrm{mL}$, did not dissolve after being subjected to light irradiation for $2 \mathrm{~h}$ (Figure 1c). Therefore, the increase of iron concentration with respect to the alginate concentration resulted in an increase of the time for gel-sol transition for light irradiated iron alginate hydrogels. For the following experiments, we chose to work with the hydrogel that presented the shortest time for sol-gel transition, Alg5_Fe4 (alginate concentration $5 \mathrm{mg} / \mathrm{mL}$ and iron concentration $4 \mathrm{mg} / \mathrm{mL}$ ). 

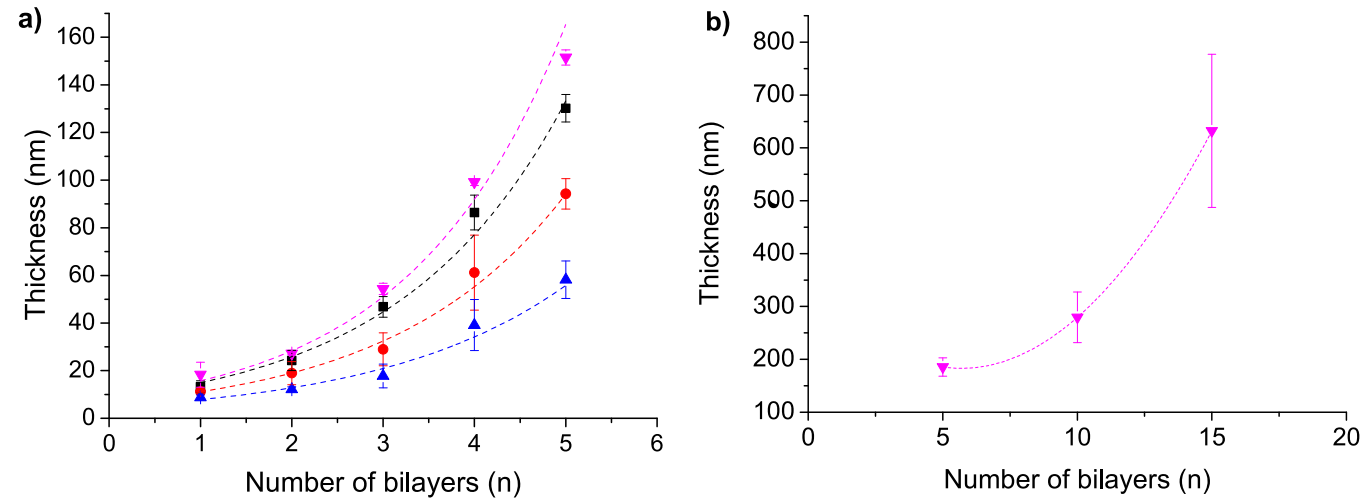

Figure 2. (a) Evolution of the thickness, measured by ellipsometry, vs the number of bilayers of (HA1.2/PAH1.0) ${ }_{n}($ red $\bullet$ ), (HA1.2/PAH2.0) (blue $\boldsymbol{\Delta}),(\mathrm{HA} 2.5 / \mathrm{PAH} 1.0)_{n}(\boldsymbol{\nabla})$, and (HA2.5/PAH2.0) ${ }_{n}$ (pink $\boldsymbol{\nabla}$ ) films. (b) Evolution of (HA2.5/PAH2.0) ${ }_{n}$ film thickness, measured by AFM, vs the number of bilayers. Dashed lines are a guide to the eye.
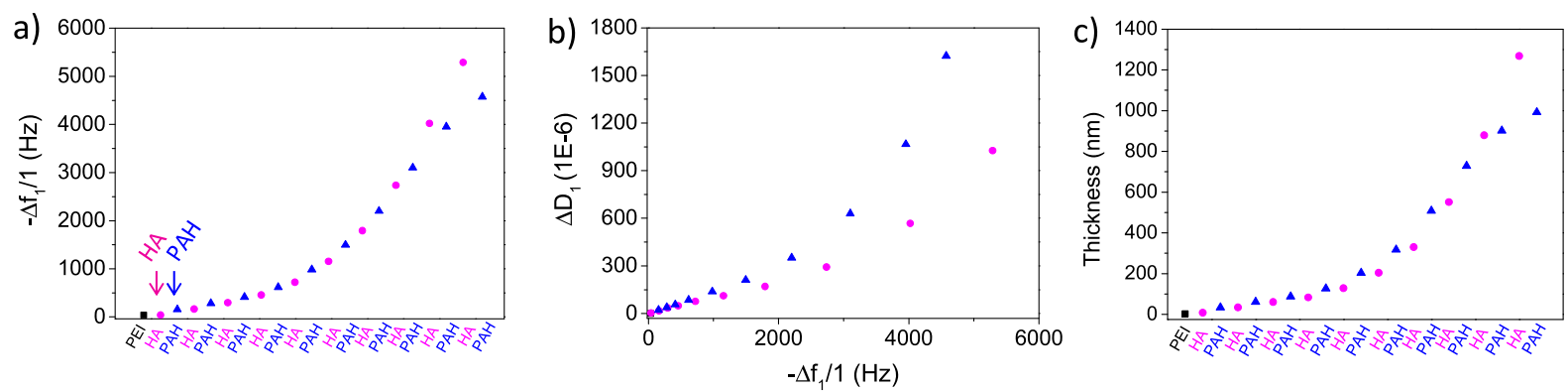

Figure 3. (a) Fundamental frequency shift, measured at $5 \mathrm{MHz}$ by QCM-D, as a function of time during the alternated depositions of HA (pink and PAH (blue $\boldsymbol{\Delta})$ using HA $(2.5 \mathrm{mg} / \mathrm{mL})$ and PAH $(2.0 \mathrm{mg} / \mathrm{mL})$. (b) Fundamental dissipation versus the fundamental frequency shift at each deposited layer of HA $(\bullet)$ and PAH $(\boldsymbol{\Delta})$. (c) Evolution of the HA/PAH film thickness, determined by fitting the experimental data with the Voigt-Voinova model, as a function of the deposited layers.
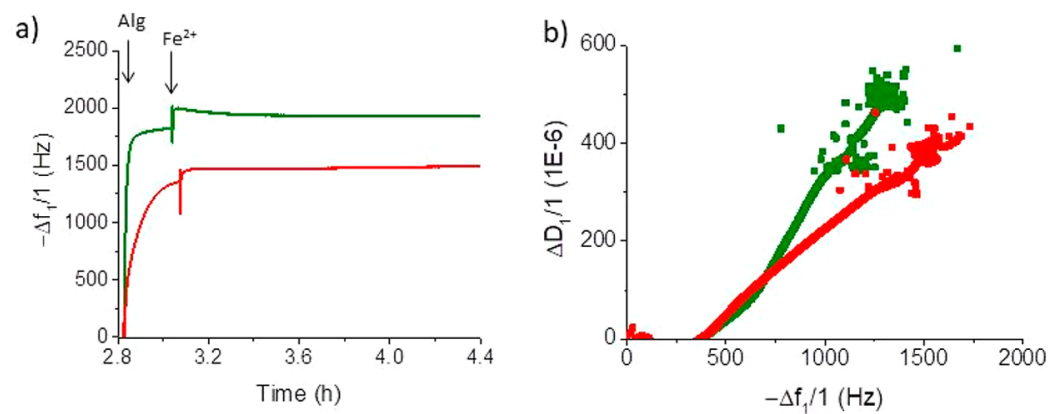

Figure 4. (a) Normalized frequency shift $\left(-\Delta f_{1} / 1\right)$, measured at $5 \mathrm{MHz}$ by QCM-D, as a function of time during the buildup of the hydrogel Alg Fe with (red line) and without lactate (green line) on top of the (HA/PAH) 10 multilayer film. (b) Fundamental dissipation versus the fundamental frequency shift with (red line) and without (green line) lactate.

Study of the Buildup of (HA/PAH) $)_{n}$ Multilayer Films. The buildup of HA/PAH multilayers was optimized using different polyelectrolyte concentrations. Figure $2 \mathrm{a}$ shows the thickness evolution with the number of bilayers, measured by ellipsometry for films prepared with $\mathrm{HA}$ and $\mathrm{PAH}$ concentrations ranging from 1 to $2.5 \mathrm{mg} / \mathrm{mL}$. The thickness of (HA/ $\mathrm{PAH})_{n}$ films increases exponentially with the number of bilayers up to five bilayers in all cases. The film thickness increase is more pronounced for films fabricated with the highest concentration of $\mathrm{HA}(2.5 \mathrm{mg} / \mathrm{mL})$. At constant $\mathrm{HA}$ concentration, the growth of films is higher for the highest $\mathrm{PAH}$ concentration tested $(2.0 \mathrm{mg} / \mathrm{mL})$. The exponential growth was attributed to the presence of $\mathrm{NaCl}$ in the assembly process because films assembled without $\mathrm{NaCl}$ showed a linear growth, as previously reported. ${ }^{40,41}$ Exponentially growing films are dynamic entities where diffusion and exchange processes of at least one of the polyelectrolytes through the entire film thickness take place at each deposition step. ${ }^{42}$ In our case, the exponential growth could be related to the diffusion of $\mathrm{PAH}$ in and out of the multilayer film at each deposition step of $\mathrm{PAH}$ and HA, respectively, as previously reported. ${ }^{43} \mathrm{~A}$ higher concentration of $\mathrm{PAH}$ provides more amine groups to diffuse into the structure, increasing the thickness. Taking into account the restrictions of ellipsometry to determine thicknesses of films with more than five bilayers, AFM performed in a dry state was used to image scratched (HA2.5/PAH2.0) ${ }_{n}$ films (Figure S2 in SI). The film thickness increases from $160 \mathrm{~nm}$ for films with five bilayers to $650 \mathrm{~nm}$ for films with 15 bilayers, thus confirming the exponential growth of (HA2.5/PAH2.0 $)_{n}$. For the following experiments, 
Scheme 2. Formation of Alg_Fe-Supported Hydrogel Built on Top of HA/PAH Multilayer Film by Deposition of Alg and Contact with $\mathrm{Fe}^{2+}$ Solution, Further Oxidized in $\mathrm{Air}^{a}$

a)

$(\mathrm{HA} / \mathrm{PAH})_{10}$

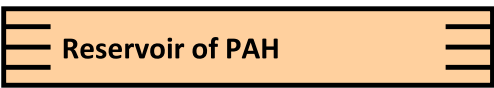

c)

$(\mathrm{HA} / \mathrm{PAH})_{10}-\mathrm{Alg} / \mathrm{Fe}^{3+}$

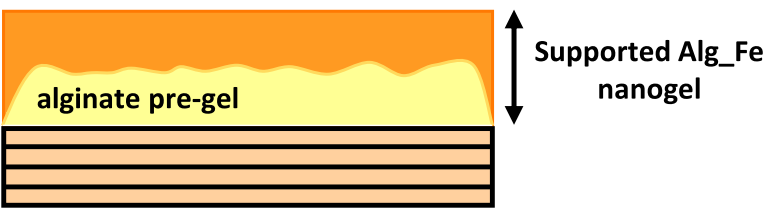

b) $(\mathrm{HA} / \mathrm{PAH})_{10}$-Alginate pre-gel

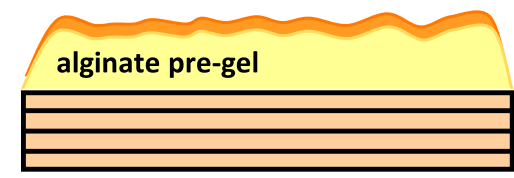

d)

$(\mathrm{HA} / \mathrm{PAH})_{10}$-Alginate pre-gel after LED illumination

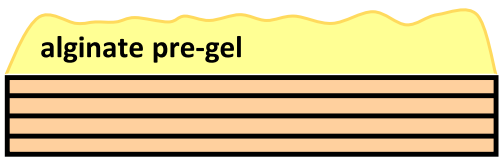

${ }^{a}$ The supported alginate hydrogel is formed by an alginate pregel obtained by a partial complexation of the alginate chains with PAH chains from the multilayer HA/PAH film and a Alg_Fe nanogel formed by the free alginate chains, which do not interact with PAH, and the iron(II) ions. Only the part of the supported hydrogel corresponding to the Alg Fe nanogel is dissolved by UV irradiation while the alginate pregel formed by complexation between $\mathrm{Alg}$ and $\mathrm{PAH}$ remains stable after UV irradiation.

(HA2.5/PAH2.0 $)_{10}$ multilayer films with a thickness of $280 \mathrm{~nm}$ were chosen as substrates for the deposition of supported iron alginate hydrogels and designated as $(\mathrm{HA} / \mathrm{PAH})_{10}$.

In Situ Study of the Buildup and Photodissolution of Supported Iron Alginate Hydrogels through QCM-D. The entire buildup of $(\mathrm{HA} / \mathrm{PAH})_{10}$ multilayer films followed by Alg_Fe hydrogel formation was monitored in situ by QCM$\mathrm{D}$ using an open cell. During the buildup of the $(\mathrm{HA} / \mathrm{PAH})_{10}$ multilayer film, the evolution of the normalized frequency shift $\left(-\Delta f_{\nu} / \nu\right)$ and the dissipation $\left(\Delta D_{\nu}\right)$ show an exponential growth in the wet state which is consistent with the results obtained in the dry state (Figure S3 in SI). Figure 3a shows the normalized frequency shift $-\Delta f_{1} / 1$ after each deposited layer, $\mathrm{HA}$ in pink and $\mathrm{PAH}$ in blue. To evaluate the rigidity properties of the film, Figure $3 \mathrm{~b}$ shows the evolution of $\Delta D_{1}$ versus $-\Delta f_{1} / 1$. The higher slope of the blue curve indicates a more rigid behavior of $\mathrm{PAH}$-ending films in comparison with HA-ending films. This could be explained by the diffusion of $\mathrm{PAH}$ inside the film, when PAH is the last layer deposited, creating a $\mathrm{PAH}$ reservoir and rendering the film more dense. When HA is the last deposited layer, PAH is diffusing out of the film to complex with HA chains, leading to less dense film. The thickness of HA/PAH film, obtained by fitting the experimental data with the Voigt-Voinova model, reaches a thickness of $1.1 \pm 0.1 \mu \mathrm{m}$ for 10 bilayers (Figure $3 \mathrm{c}$ ).

After $(\mathrm{HA} / \mathrm{PAH})_{10}$ film buildup, a layer of $\mathrm{Alg}(5 \mathrm{mg} / \mathrm{mL})$ was deposited onto the multilayer film (with and without sodium lactate) followed by contact with an aqueous solution of $\mathrm{Fe}^{2+}$ cations (Figure 4a). In both cases, a high increase of $-\Delta f_{1} / 1, \sim 1360 \mathrm{~Hz}$, is obtained after the deposition of alginate and a smaller one, $\sim 100 \mathrm{~Hz}$, after the injection of the aqueous solution containing $\mathrm{Fe}^{2+}$ cations. The contact with alginate solution leads to a high frequency shift due to the complexation of alginate chains with PAH chains. Indeed, as in the case of HA deposition, PAH is diffusing out of the film to complex with alginate chains, leading to the formation of $\mathrm{PAH} / \mathrm{Alg}$ complexes. Further contact with $\mathrm{Fe}^{2+}$ cations gives rise to a smaller increase in frequency shift which can indicate their complexation with alginate chains. In the presence of sodium lactate, the frequency shift signal slightly increases with the time due to the oxidation of "soft" $\mathrm{Fe}^{2+}$ to "hard" $\mathrm{Fe}^{3+}$ in contact with air giving rise to a hydrogel (zoom-in Figure S4 in $\mathrm{SI}$ ). The influence of sodium lactate in the rigidity of the hydrogel was studied by QCM-D. Figure $4 \mathrm{~b}$ exhibits the variation of $\Delta D_{1}$ versus $-\Delta f_{1} / 1$ for hydrogels prepared with and without sodium lactate. The slope of the curve is lower in the presence of lactate indicating a lower rigidity of Alg_Fe gel (Figure $4 b$ ). This result is consistent with the elastic modulus, determined through oscillatory shear measurements, measured for bulk Alg_Fe hydrogels prepared at the same Alg and $\mathrm{Fe}^{2+}$ concentrations $(5 \mathrm{mg} / \mathrm{mL}$ alginate concentration and $4 \mathrm{mg} /$ $\mathrm{mL}$ iron concentration) (Figure S5 in SI). The value of the elastic moduli measured at $1 \mathrm{~Hz}$ frequency for Alg_Fe is $100 \pm$ $8 \mathrm{~Pa}$. Lactate addition decreases the elastic moduli of the resulting gels to $65 \pm 5 \mathrm{~Pa}$ yet preserving the gel formation as demonstrated through oscillatory frequency tests. This could be due to competitive complexation of $\mathrm{Fe}^{2+}$ with sodium lactate carboxylic groups thus preventing the interaction with alginate carboxylic groups. To determine the thickness of the supported iron alginate gel with incorporated sodium lactate fabricated on top of the multilayer $(\mathrm{HA} / \mathrm{PAH})_{10}$ film, experimental data from QCM-D were fitted by using the Voigt-Voinova model. The thickness obtained for the supported alginate gel is $437 \pm 231 \mathrm{~nm}$ before the addition of the aqueous solution of $\mathrm{Fe}^{2+}$ cations and increases slightly to $490 \pm 215 \mathrm{~nm}$ with the addition of $\mathrm{Fe}^{2+}$. These results highlight the fact that the main composition of the supported gel is $\mathrm{PAH} / \mathrm{Alg}$ complexes.

Scheme 2 represents the proposed mechanism for the formation of Alg_Fe hydrogel supported on a HA/PAH multilayer film. $(\overline{\mathrm{H}} \mathrm{A} / \mathrm{PAH})_{10}$ film constitutes a reservoir of $\mathrm{PAH}$ chains, due to the exponential growing behavior of the film (Scheme 2a). The contact of the alginate solution with this film leads to a partial complexation of the alginate chains 

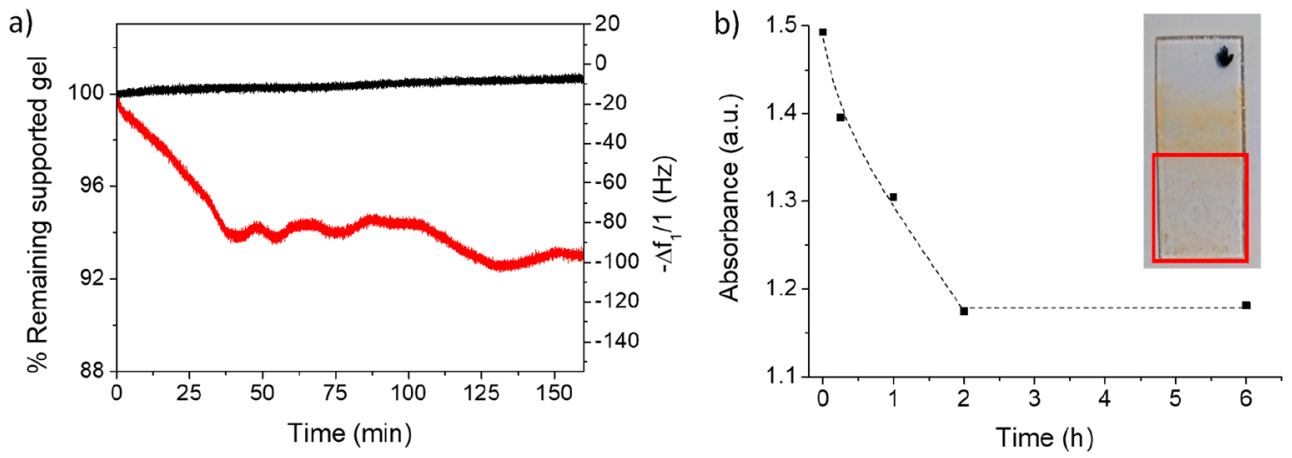

Figure 5. (a) Percent remaining supported gel and normalized frequency shift $\left(-\Delta f_{1} / 1\right)$, measured at $5 \mathrm{MHz}$ by QCM-D, as a function of time for the supported iron alginate hydrogels in contact with water non-light-irradiated (black line) and light-irradiated (red line). (b) Evolution of the UV-vis absorbance, measured at $236 \mathrm{~nm}$, as a function of time of the supported iron alginate hydrogel exposed to light from LED source. The dashed line is a guide to the eye. The inset shows a photo of the supported hydrogel after being immersed for $6 \mathrm{~h}$ in water. The red rectangle marks the area subjected to light irradiation.

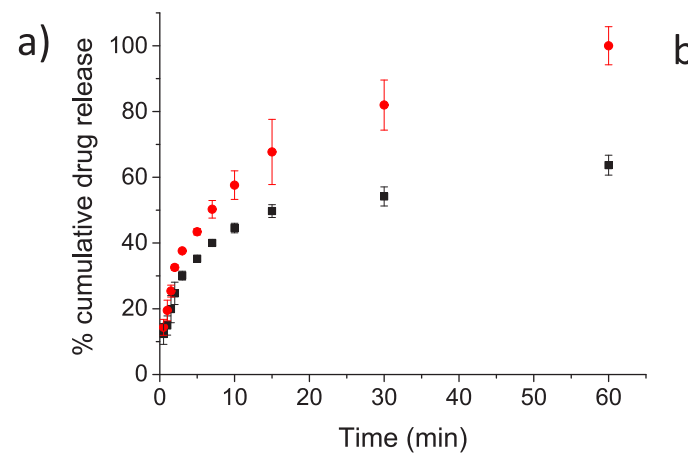

b)

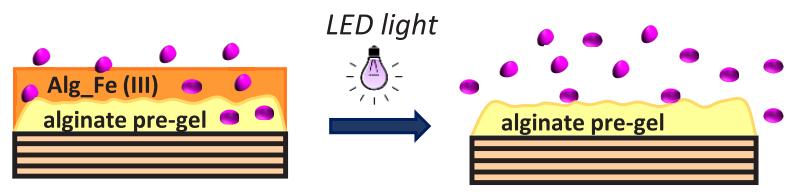

Figure 6. Release of folic acid in water from (a) supported iron alginate hydrogels non-irradiated (black points) and light-irradiated (red points) and (b) schematic representation of the mechanism proposed for the folic acid release for nonirradiated supported iron alginate hydrogels (left) and for light irradiated hydrogels (right).

with PAH chains forming a "pregel" and the presence of free alginate chains on top of the "pregel" (Scheme $2 b$ ). When the architecture is put into contact with $\mathrm{Fe}^{2+}$ ions solution, free alginate chains are complexed by "soft" $\mathrm{Fe}^{2+}$ ions and subsequently oxidized to "hard" $\mathrm{Fe}^{3+}$ ions in the presence of air, giving rise to the supported iron alginate hydrogel (Scheme 2c) with a slight increase of the obtained thickness. The proposed formation mechanism for $\mathrm{Alg} F \mathrm{Fe}$ hydrogels is analogous to that described for $\mathrm{Alg} \mathrm{Ca}^{2+}$ gels built on top of PLL/HA exponentially growing multilayers. ${ }^{44}$ Light exposure $(\lambda=405 \mathrm{~nm})$ leads to gel-sol transition of the supported hydrogel (Scheme $2 \mathrm{~d}$ ). Bruchet et al. ${ }^{45}$ have reported a partial photochemical dissolution of iron alginate hydrogels in bulk by creating a diffusion gradient of lactate through the hydrogel. In that case, a solution of lactate was put in contact with the top of the iron alginate hydrogel to be then irradiated with UV light, allowing the etching of the thin layer of hydrogel, which was in contact with lactate, while the rest of the gel without contact with lactate remained stable. In our case, we follow a different strategy by creating a supported hydrogel composed of an alginate pregel with sodium lactate incorporated, which is not dissolved by UV irradiation due to its complexation with free $\mathrm{PAH}$ chains from the multilayer film $\mathrm{HA} / \mathrm{PAH}$ acting as a support, and a Alg_Fe nanogel over it, which is phototriggered by UV irradiation.

The gel-sol transition of light-irradiated iron alginate hydrogels was followed by QCM-D by placing the LED source on the top of the open cell (Figure 5a). For non- irradiated hydrogels, $-\Delta f_{1} / 1$ is constant during the entire experimental time, showing stability of the hydrogel in contact with water for at least $2 \mathrm{~h}$ (100\% of remaining gel). In contrast, under irradiation, the supported hydrogel loses $7 \%$ in frequency shift in $2 \mathrm{~h}$. The fundamental frequency shift of the iron alginate hydrogel decreases $-\Delta f_{1} / 1 \sim-100 \mathrm{~Hz}$ over an initial value of $-1460 \mathrm{~Hz}$. This dissolved part of the supported gel is the one formed after the addition of $\mathrm{Fe}^{2+}$ (Figure 4a). Most of the supported hydrogel (93\%) is stable in contrast to the bulk gel which is completely destroyed after 65 min (Figure 1a).

The photodissolution of the supported hydrogel was also ascertained through UV-vis spectroscopy (Figures 5b and S5a in SI). For that purpose, the whole sample was immersed in water and only the area marked by a red rectangle was placed under LED source for $6 \mathrm{~h}$. Outside of the red rectangle, the supported hydrogel keeps its light orange color characteristic of the presence of $\mathrm{Fe}^{3+}$ inside the gel (Figure 5b). There is no change detected in the UV-visible spectra over time (Figure $\mathrm{S} 6 \mathrm{~b}$ in SI). The supported hydrogel area subjected to light irradiation loses its light orange color (red rectangle in Figure $5 b)$. A decrease of the absorbance at $236 \mathrm{~nm}$ is observed over time until reaching a steady state after $2 \mathrm{~h}$. This is due to the photoreduction of $\mathrm{Fe}^{3+}$ into $\mathrm{Fe}^{2+}$ triggered by light.

Phototriggered Drug Release. Folic acid was employed as a model drug to demonstrate the concept of phototriggered drug release. The results corresponding to cumulative drug 
release of folic acid from supported alginate hydrogels with and without light are depicted in Figure 6.

For non-irradiated supported hydrogels, $\sim 60 \%$ folic acid was released within $60 \mathrm{~min}$ and $\sim 100 \%$ of folic acid was released within $24 \mathrm{~h}$. Light irradiation of iron alginate hydrogels greatly accelerates folic acid release, and $\sim 80 \%$ folic acid was released within $30 \mathrm{~min}$ (Figure 6a). Folic acid release profiles from bulk hydrogels (Figure S7 in SI) present a similar trend, that is, light irradiation accelerates folic acid release; $\sim 15 \%$ folic acid was released within $15 \mathrm{~min}$ for non-irradiated alginate hydrogels and increases to $\sim 100 \%$ folic acid release for light-irradiated alginate hydrogels.

The results obtained for supported alginate hydrogels can be correlated to those obtained by QCM-D (Figure 5a) which shows that supported iron alginate hydrogels remain stable in contact with water with no notable dissolution in the absence of light exposure. In contrast, light irradiation leads to the dissolution of $80 \%$ of the supported hydrogel in $30 \mathrm{~min}$ which leads to an increased rate of folic acid release. The results can be further explained, taking into account the two phases proposed for iron alginate hydrogels depicted in Figure 6b: $\mathrm{Alg} / \mathrm{Fe}^{3+}$ gel and the alginate pregel. In the absence of light, $\mathrm{Alg} / \mathrm{Fe}$ complexes have high interaction with $\mathrm{Fe}^{3+}$, giving rise to low release of folic acid throughout this gel layer (bottom). With light, the absence of the $\mathrm{Alg} / \mathrm{Fe}^{3+}$ gel due to its phototriggered gel-sol transition leads to a faster folic acid release.

A comparison of the release profiles obtained from supported and bulk hydrogels allows us to conclude that for non-irradiated hydrogels, the initial drug release (within the first $10 \mathrm{~min}$ ) was accelerated for supported alginate hydrogels with respect to bulk alginate hydrogels. This might be attributed to the fact that quick diffusion of folic acid from the surface layer of the nanometer-scale iron alginate hydrogels might play a dominant role during the initial drug release period. ${ }^{46}$ Conversely, light-irradiated supported hydrogels show a more sustained folic acid release over time with respect to bulk hydrogels due to the presence of an alginate pregel consisting of $\mathrm{PAH} / \mathrm{Alg}$ complexes that hinders the burst release of folic acid seen in bulk alginate hydrogels.

\section{CONCLUSIONS}

Nanometer-scale alginate hydrogels have been formed on top of a multilayer HA/PAH film employing the dipping-assisted LbL technique. The buildup process has been followed in situ by QCM-D, allowing us to determine the gel thickness, $\sim 490$ $\mathrm{nm}$, and the influence of sodium lactate on the elastic properties of the gel. The iron and alginate concentrations needed for the formation of bulk iron alginate hydrogels and the time for phototriggered gel-sol transition were previously determined by inverted tube tests. In addition, the optimal experimental parameters for the buildup of $\mathrm{HA} / \mathrm{PAH}$ multilayer films, in terms of the number of bilayers and concentration of $\mathrm{HA}$ and $\mathrm{PAH}$, were determined by ellipsometry and AFM. The phototriggered dissolution of the supported hydrogels through $\mathrm{Fe}^{3+}$ reduction into $\mathrm{Fe}^{2+}$ was followed by QCM-D and UV-vis spectroscopy, showing a complete dissolution of the supported alginate hydrogel after light exposure for $2 \mathrm{~h}$. The potential application of the supported nanometer-scale hydrogels for phototriggered drug release was proven by incorporation of folic acid during gel formation and subsequent release triggered by light from an LED source. With light irradiation, the design of supported nanometer-scale photoresponsive alginate hydrogels constitutes a strategy for a more sustained drug delivery over time with respect to bulk alginate hydrogels.

\section{ASSOCIATED CONTENT}

\section{Supporting Information}

The Supporting Information is available free of charge on the ACS Publications website at DOI: 10.1021/acs.langmuir.9b01224.

AFM topographic images corresponding to (HA/PAH) films; QCM-D data for (HA2.5/PAH2.0 $)_{10}$ and for the nanogel Alg_Fe on top; oscillatory shear measurements for bulk iron alginate hydrogels; UV-vis spectra of $(\mathrm{HA} / \mathrm{PAH})_{10}$-Alg Fe in contact with water and/or LED irradiation; release of folic acid from bulk iron alginate hydrogels (PDF)

\section{AUTHOR INFORMATION}

\section{Corresponding Authors}

*E-mail: rhernandez@ictp.csic.es. Tel: +34 915622900 (ext 921504) (R.H.).

*E-mail: mcriado@ictp.csic.es. Tel: +34 915622900 (M.C.G.).

ORCID $\odot$

Miryam Criado-Gonzalez: 0000-0002-5502-892X

Fouzia Boulmedais: 0000-0002-4934-9276

Rebeca Hernández: 0000-0001-7332-0134

\section{Notes}

The authors declare no competing financial interest.

\section{ACKNOWLEDGMENTS}

The authors thank Emilio Madrid for rheological measurements and Pilar Posadas for AFM measurements. Déborah Wagner is acknowledged for technical support. M.C.-G. acknowledges IDEX of Université de Strasbourg for granting a postdoctoral fellowship. Financial support from MINECO (MAT2017-83014-C2-1-P) is gratefully acknowledged.

\section{REFERENCES}

(1) Criado, M.; Sanz, B.; Goya, G. F.; Mijangos, C.; Hernandez, R. Magnetically responsive biopolymeric multilayer films for local hyperthermia. J. Mater. Chem. B 2017, 5 (43), 8570-8578.

(2) Zamora-Mora, V.; Fernández-Gutiérrez, M.; González-Gómez, Á.; Sanz, B.; San Román, J.; Goya, G. F.; Hernández, R.; Mijangos, C. Chitosan nanoparticles for combined drug delivery and magnetic hyperthermia: From preparation to in vitro studies. Carbohydr. Polym. 2017, 157, 361-370.

(3) Hanuš, J.; Ullrich, M.; Dohnal, J.; Singh, M.; Štěpánek, F. Remotely Controlled Diffusion from Magnetic Liposome Microgels. Langmuir 2013, 29 (13), 4381-4387.

(4) Hernández, R.; Criado, M.; Nogales, A.; Sprung, M.; Mijangos, C.; Ezquerra, T. A. Deswelling of Poly(N-isopropylacrylamide) Derived Hydrogels and Their Nanocomposites with Iron Oxide Nanoparticles As Revealed by X-ray Photon Correlation Spectroscopy. Macromolecules 2015, 48 (2), 393-399.

(5) Tekin, H.; Sanchez, J. G.; Tsinman, T.; Langer, R.; Khademhosseini, A. Thermoresponsive Platforms for Tissue Engineering and Regenerative Medicine. AIChE J. 2011, 57 (12), 32493258.

(6) Pastuszka, M. K.; MacKay, J. A. Engineering structure and function using thermoresponsive biopolymers. Wiley Interdiscip. Rev. Nanomed. Nanobiotechnol. 2016, 8 (1), 123-138. 
(7) Mercado, S. A.; Slater, N. K. H. The functional and structural effects of an amphipathic $\mathrm{pH}$ responsive biopolymer: A comprehensive study in osteosarcoma cells. Eur. Polym. J. 2016, 74, 158-167.

(8) Burke, S. E.; Barrett, C. J. pH-Responsive Properties of Multilayered Poly(1-lysine)/Hyaluronic Acid Surfaces. Biomacromolecules 2003, 4 (6), 1773-1783.

(9) Narayanan, R. P.; Melman, G.; Letourneau, N. J.; Mendelson, N. L.; Melman, A. Photodegradable Iron(III) Cross-Linked Alginate Gels. Biomacromolecules 2012, 13 (8), 2465-2471.

(10) Tomatsu, I.; Peng, K.; Kros, A. Photoresponsive hydrogels for biomedical applications. Adv. Drug Delivery Rev. 2011, 63 (14), $1257-1266$.

(11) Shukla Sudheesh, K.; Tiwari, A. Stimuli-Responsive Redox Biopolymers. In Responsive Materials and Methods: State-of-the-Art Stimuli-Responsive Materials and Their Applications; Tiwari, A.; Kobayashi, H., Eds.; Wiley, 2013; pp 357-375.

(12) Zahouani, S.; Chaumont, A.; Senger, B.; Boulmedais, F.; Schaaf, P.; Jierry, L.; Lavalle, P. Stretch-Induced Helical Conformations in Poly(l-lysine)/Hyaluronic Acid Multilayers. ACS Appl. Mater. Interfaces 2016, 8 (24), 14958-14965.

(13) Rios, C.; Longo, J.; Zahouani, S.; Garnier, T.; Vogt, C.; Reisch, A.; Senger, B.; Boulmedais, F.; Hemmerle, J.; Benmlih, K.; Frisch, B.; Schaaf, P.; Jierry, L.; Lavalle, P. A new biomimetic route to engineer enzymatically active mechano-responsive materials. Chem. Commun. 2015, 51 (26), 5622-5625.

(14) Lee, K. Y.; Mooney, D. J. Alginate: properties and biomedical applications. Prog. Polym. Sci. 2012, 37 (1), 106-126.

(15) Liu, T.-Y.; Hu, S.-H.; Liu, T.-Y.; Liu, D.-M.; Chen, S.-Y. Magnetic-Sensitive Behavior of Intelligent Ferrogels for Controlled Release of Drug. Langmuir 2006, 22 (14), 5974-5978.

(16) Gaharwar, A. K.; Peppas, N. A.; Khademhosseini, A. Nanocomposite hydrogels for biomedical applications. Biotechnol. Bioeng. 2014, 111 (3), 441-453.

(17) Buenger, D.; Topuz, F.; Groll, J. Hydrogels in sensing applications. Prog. Polym. Sci. 2012, 37 (12), 1678-1719.

(18) Ionov, L. Biomimetic Hydrogel-Based Actuating Systems. Adv. Funct. Mater. 2013, 23 (36), 4555-4570.

(19) Gracia, R.; Mecerreyes, D. Polymers with redox properties: materials for batteries, biosensors and more. Polym. Chem. 2013, 4 (7), 2206-2214.

(20) Niaounakis, M. 1 - Definitions of Terms and Types of Biopolymers. In Biopolymers: Applications and Trends; William Andrew Publishing: Oxford, 2015; pp 1-90.

(21) Moe, S.; Draget, K. I.; Skjåk-Bræk, G.; Smidsrod, O. Alginates. In Food Polysaccharides and Their Applications; Stephen, A. M.; Phillips, G. O.; Williams, P. A., Eds.; CRC Press: New York, 2006; pp 289-334.

(22) Hernández, R.; Sacristán, J.; Mijangos, C. Sol/Gel Transition of Aqueous Alginate Solutions Induced by $\mathrm{Fe} 2+$ Cations. Macromol. Chem. Phys. 2010, 211 (11), 1254-1260.

(23) Guo, J.; Jourdian, G. W.; Maccallum, D. K. Culture and Growth Characteristics of Chondrocytes Encapsulated in Alginate Beads. Connect. Tissue Res. 1989, 19 (2-4), 277-297.

(24) Finotelli, P. V.; Morales, M. A.; Rocha-Leão, M. H.; BaggioSaitovitch, E. M.; Rossi, A. M. Magnetic studies of iron(III) nanoparticles in alginate polymer for drug delivery applications. Mater. Sci. Eng., C 2004, 24 (5), 625-629.

(25) Agulhon, P.; Markova, V.; Robitzer, M.; Quignard, F.; Mineva, T. Structure of Alginate Gels: Interaction of Diuronate Units with Divalent Cations from Density Functional Calculations. Biomacromolecules 2012, 13 (6), 1899-1907.

(26) Barrett, D. G.; Fullenkamp, D. E.; He, L.; Holten-Andersen, N.; Lee, K. Y. C.; Messersmith, P. B. pH Based Regulation of Hydrogel Mechanical Properties Through Mussel Inspired Chemistry and Processing. Adv. Funct. Mater. 2013, 23 (9), 1111-1119.

(27) Giammanco, G. E.; Sosnofsky, C. T.; Ostrowski, A. D. LightResponsive Iron(III)-Polysaccharide Coordination Hydrogels for Controlled Delivery. ACS Appl. Mater. Interfaces 2015, 7 (5), 3068-3076.
(28) Pearson, R. G. Hard and Soft Acids and Bases. J. Am. Chem. Soc. 1963, 85 (22), 3533-3539.

(29) Jin, Z.; Güven, G.; Bocharova, V.; Halámek, J.; Tokarev, I.; Minko, S.; Melman, A.; Mandler, D.; Katz, E. Electrochemically Controlled Drug-Mimicking Protein Release from Iron-Alginate ThinFilms Associated with an Electrode. ACS Appl. Mater. Interfaces 2012, 4 (1), 466-475.

(30) Peng, F.; Li, G.; Liu, X.; Wu, S.; Tong, Z. Redox-Responsive Gel-Sol/Sol-Gel Transition in Poly(acrylic acid) Aqueous Solution Containing Fe(III) Ions Switched by Light. J. Am. Chem. Soc. 2008, 130 (48), 16166-16167.

(31) He, S.; Ren, B.; Liu, X.; Tong, Z. Reversible Electrogelation in Poly(acrylic acid) Aqueous Solutions Triggered by Redox Reactions of Counterions. Macromol. Chem. Phys. 2010, 211 (23), 2497-2502.

(32) Criado, M.; Rebollar, E.; Nogales, A.; Ezquerra, T. A.; Boulmedais, F.; Mijangos, C.; Hernández, R. Quantitative Nanomechanical Properties of Multilayer Films Made of Polysaccharides through Spray Assisted Layer-by-Layer Assembly. Biomacromolecules 2017, 18 (1), 169-177.

(33) Criado-Gonzalez, M.; Fernandez-Gutierrez, M.; San Roman, J.; Mijangos, C.; Hernández, R. Local and controlled release of tamoxifen from multi (layer-by-layer) alginate/chitosan complex systems. Carbohydr. Polym. 2019, 206, 428-434.

(34) Park, K.; Choi, D.; Hong, J. Nanostructured Polymer Thin Films Fabricated with Brush-based Layer-by-Layer Self-assembly for Site-selective Construction and Drug release. Sci. Rep. 2018, 8 (1), 3365.

(35) Anandhakumar, S.; Raichur, A. M. Polyelectrolyte/silver nanocomposite multilayer films as multifunctional thin film platforms for remote activated protein and drug delivery. Acta Biomater. 2013, 9 (11), 8864-8874.

(36) King, K. Changes in the functional properties and molecular weight of sodium alginate following $\gamma$ irradiation. Food Hydrocolloids 1994, 8 (2), 83-96.

(37) Lavalle, P.; Senger, B.; Schaaf, P. Surface Methods. In Nanoscience: Nanobiotechnology and Nanobiology; Boisseau, P.; Houdy, P.; Lahmani, M., Eds.; Springer: Berlin, 2009; pp 561-577.

(38) Sima, J.; Makáňová, J. Photochemistry of iron (III) complexes. Coord. Chem. Rev. 1997, 160, 161-189.

(39) Küpper, F. C.; Carrano, C. J.; Kuhn, J.-U.; Butler, A. Photoreactivity of Iron(III)-Aerobactin: Photoproduct Structure and Iron(III) Coordination. Inorg. Chem. 2006, 45 (15), 6028-6033.

(40) Pettersson, T. r.; Pendergraph, S. A.; Utsel, S.; Marais, A.; Gustafsson, E.; Wågberg, L. Robust and Tailored Wet Adhesion in Biopolymer Thin Films. Biomacromolecules 2014, 15 (12), 44204428.

(41) Szarpak, A.; Pignot-Paintrand, I.; Nicolas, C.; Picart, C.; AuzélyVelty, R. Multilayer assembly of hyaluronic acid/poly (allylamine): control of the buildup for the production of hollow capsules. Langmuir 2008, 24 (17), 9767-9774.

(42) Ball, V.; Hübsch, E.; Schweiss, R.; Voegel, J.-C.; Schaaf, P.; Knoll, W. Interactions between Multivalent Ions and Exponentially Growing Multilayers: Dissolution and Exchange Processes. Langmuir 2005, 21 (18), 8526-8531.

(43) Szarpak, A.; Cui, D.; Dubreuil, F. d. r.; De Geest, B. G.; De Cock, L. J.; Picart, C.; Auzely-Velty, R. Designing hyaluronic acidbased layer-by-layer capsules as a carrier for intracellular drug delivery. Biomacromolecules 2010, 11 (3), 713-720.

(44) Mjahed, H.; Porcel, C.; Senger, B.; Chassepot, A.; Netter, P.; Gillet, P.; Decher, G.; Voegel, J.-C.; Schaaf, P.; Benkirane-Jessel, N.; Boulmedais, F. Micro-stratified architectures based on successive stacking of alginate gel layers and poly(1-lysine)-hyaluronic acid multilayer films aimed at tissue engineering. Soft Matter 2008, 4 (7), $1422-1429$.

(45) Bruchet, M.; Mendelson, L. N.; Melman, A. Photochemical Patterning of Ionically Cross-Linked Hydrogels. Processes 2013, 1 (2), 153.

(46) Liu, G.; Dong, C.-M. Photoresponsive Poly(S-(o-nitrobenzyl)-1cysteine)-b-PEO from a l-Cysteine $\mathrm{N}$-Carboxyanhydride Monomer: 
Synthesis, Self-Assembly, and Phototriggered Drug Release. Biomacromolecules 2012, 13 (5), 1573-1583. 